\title{
Knowledge, Practice and Barriers of Foot Self-Care among Diabetic Patients at Tanta University Hospitals, Egypt
}

\author{
Mira M. Abu-elenin ${ }^{1}$, Ahmed A. Elshoura ${ }^{2}$, Ghada M.Alghazaly ${ }^{3}$ \\ ${ }^{1}$ Community Medicine Department, ${ }^{2}$ General Surgery Department, ${ }^{3}$ Internal Medicine \\ Department, Faculty of Medicine, Tanta Uiversity.
}

Received: March, 2018 Accepted: May, 2018

\begin{abstract}
Background : Diabetes related foot disease is a major cause of morbidity and mortality in diabetic patients. Interventions to reduce the burden of diabetic foot disease are highly cost effective. An estimated $15 \%$ of patients with diabetes develop foot ulcer. Good knowledge and practice regarding diabetic foot care will reduce the risk of diabetic foot complications and ultimately amputation. Objective: This study is conducted to assess patients' knowledge, practice and barriers of foot self-care among diabetic patients attending Tanta University Hospitals. Methods: A descriptive cross-sectional study was conducted in 2016, included 264 diabetic patients attending outpatients clinics at Tanta University hospitals. Data was collected through interviewing patients using a questionnaire based on recommendations of the American Diabetes Association. Results: $42.0 \%$ of the participants were males and 58.0\% were females. Half of them had good knowledge level $(51 \%)$, while $(62.2 \%)$ had inadequate self-foot care practice. There was a significant association between level of education with the level of diabetic foot care knowledge and practice. Poor communication between patients and health care providers (54.7\%) and lack of adequate knowledge $(50.2 \%)$ were the most reported barriers. Conclusion: The overall practice score of studied patients, was inadequate and they were not adequately caring for their feet in spite of their good knowledge.
\end{abstract}

Keywords: Diabetic foot, Self-care, Knowledge, Practice

Corresponding Author: Dr.Mira Maged Mohamed Abu-elenin Email: mira.maged@ hotmail.com

\section{Introduction}

Diabetic foot ulcers (DFU) contribute significantly to the morbidity and mortality of patients with diabetes mellitus. ${ }^{1}$ Diabetic foot ulcer (DFU) is a fullthickness wound penetrating through the dermis, below the ankle in a diabetic patient. If not treated, it may become infected. $^{2}$

DFU result from many risk factors such as; long duration of diabetes $\geq 10$ years, poor glucose control, older age, peripheral vasculopathy and poor knowledge of selfcare. ${ }^{3}$ Increasing urbanization leads to unhygienic conditions, barefoot gait, low income, and cultural practices. In Egypt, there is scarcity of data on prevalence of such risk factors. ${ }^{4}$

The risk of developing diabetic foot ulceration is $10-15 \%$. Most of foot ulcers (60-80\%) will heal, while $10-15 \%$ of them will remain active, and 5-24\% of them will end with limb amputation within of 6-18 months. ${ }^{5}$ Every 30 seconds a lower limb is lost somewhere in the world as a 
consequence of diabetes. ${ }^{6,7}$ In the United States, about $60 \%$ of non-injured lower extremity amputation (LEA) results from DM. After a first LEA, about half of patients require another amputation within 3-5 years. ${ }^{8}$

Other adverse effects of DFU include physical disability, poor health related quality of life and economic burden ${ }^{(6,7)}$. Foot ulcers often reoccur even after healing, thus; prevention of DFU is of extreme importance. ${ }^{9}$

Regular foot examination is necessary to identify risky patients and to implement timely appropriate interventions. Special callus and nail care, wound care, and patient education should be provided by a multi-disciplinary team. But, it is difficult to be implemented into primary health care services. ${ }^{10}$

The center for disease control and prevention (CDC) reported that complementary foot-care programs including risk assessment, self foot-care education , metabolic control, and referral to specialists may decrease the risk of amputation by $45 \%-85 \%$ (12) . Those measures are highly cost-effective and cost saving. ${ }^{13}$

In Arab countries, diabetic foot management is costly. For this reason, the health care providers are somewhat reluctant to admit patients with diabetic foot problems, besides physical and social rehabilitation is still an underdeveloped field. $^{14}$

In Egypt, in 2015 there were over 7.8 million cases of diabetes, the prevalence rate among adult was $17.9 \%$, and the annual cost per person is 218.8 USD. $^{15}$ Progress needs to be made in national plans of preventive policies and to increase funding for cost-effective diabetes prevention and management of complication. ${ }^{16}$ This research aimed to study the current status of knowledge, practices and barriers towards foot care among Egyptian diabetic patients seeking care at Tanta University Hospitals.

\section{Methods}

A hospital based descriptive crosssectional study was conducted from February to April, 2016. The study was conducted at Tanta University Hospitals in Tanta city, which is the capital city of ElGharbya Governorate in middle Delta region of Egypt. It is $92 \mathrm{Km}$ north Cairo.

The total population served by the hospital is about 17 million. A total sample of 264 diabetic patients were recruited from both outpatient Internal Medicine and General Surgery department using systematic random sampling technique. Study participants were consecutively interviewed till final sample sized was reached.

The sample size was determined using Epiinfo program assuming that the prevalence of good knowledge of foot care is $50 \%$ (since no similar study was found in the area) with $95 \%$ confidence interval and $80 \%$ the power of the study.

Consented patients diagnosed of type I and II diabetes for at least six months were included in the study. The questionnaire consisted of three parts. Part I include sociodemographic data and medical history, part II : 12 questions related to knowledge of foot care, part III:15 question regarding self-care practice. This tool was adapted from previous study in India, ${ }^{7}$ and it is based on recommendation of the American College of Foot and Ankle Surgeons and the American Diabetes Association ${ }^{(17,18)}$. The designed questionnaire was tested for its validity and reliability, Alpha Cronbach`s reliability was 0.86 . Statistical analysis: after compilation of collected data, analysis was done using Statistical Package for Social Sciences (SPSS), version 20 (IBM, Chicago, USA). 
Quantitative date was presented as mean and standard deviation. Number and percentage were used to represent qualitative data, Chi Square test was the test of significance, when it is not appropriate Monte Carlo Exact test was used instead .Multinomial logistic regression analysis modelling was used in analyzing factors affecting diabetic foot care practice ,

Ethical consideration: An ethical approval was obtained from the ethics committee in Tanta Faculty of Medicine, Egypt, before the enrolment of patients started. A free informed consent was obtained from all patients.

\section{Results}

A total of 264 diabetes patients were involved in the study with the response rate of $100 \%$, as consecutive respondents were recruited until sample size was reached. Among the total of 264 respondents, $111(42.0 \%)$ were males and $153(58.0 \%)$ were females. The mean age was $53.44( \pm 13.75)$ years ranged from $(18$ $88)$ and about third of the patients (34.1\%) lied between 46 and 55 years. The participants were nearly equally distributed from rural and urban residence. The result on educational level showed that a large proportion of participants 129 (49.0\%) were low educated (illiterate, primary and preparatory).

The majority of the respondents were housewives 115 (43.0\%), and the least had professional jobs. Furthermore, substantial proportion $157(59.5 \%)$ mentioned that their income was quietly enough.

Medical History of Study Participants: The majority of study participant $(77.3 \%)$ had type II DM and the reported mean age of DM onset was at 41.3 years old, ranged from 10 to 73 .The mean duration of DM in years was $11.3 \pm 8.6$ years, and the majority of respondents $205(77.7 \%)$ live with diabetes for more than 5 years .

The mean fasting blood sugar was 255.47 $\pm 91 \mathrm{mg} / \mathrm{dl}$ ranged from 100-600 mg/dl. Regarding history of chronic diseases, half of the patients had history of hypertension $134(50.8 \%)$.

Knowledge and Practice regarding Diabetic Foot Care:The knowledge responses for each foot care measure were generally good except for some areas related for inspection of feet and inside the foot wear $(50.8 \%, 49.2 \%$ respectively) ,also washing technique and skin care of the feet in case of redness/bleeding between toes $(50.8 \%, 44.3 \%$ respectively ) as shown in (Table 1).

Negative responses for foot care practice were high in items related to regular inspection of feet $(44.3 \%)$, adding skin emollient to feet $(62.0 \%)$, checking shoes when putting on $(48.5 \%)$ nor taking off $(60.6 \%)$.More than half of them didn't receive any advice about the type of foot wear and $49.6 \%$ regularly walk bared foot indoors.(Table 2)

Upon analyzing sociodemographic data in relation to diabetic foot care knowledge and practice ,the present research showed that highly educated patients were significantly had good knowledge and practice level and most of type II DM patients had bad self-foot care practice.

It is worthy to mention that level of selfcare practice was significantly associated with level of knowledge, where $88 \%$ of patient with poor knowledge, had inadequate foot self-care practice (Figure $1)$.

All sociodemographic characteristics were tested with the categorized practice score of foot care using a multinomial logistic regression analysis (table 3 ), it showed that patients with history of diabetic foot ulceration were three times more likely to do better foot self-care practice $(\mathrm{p}=0.04)$ 
Table 1: Percentage distribution of knowledge responses regarding diabetic foot care.

No. Questions related to knowledge of foot care

\begin{tabular}{|l|l|}
\hline $\begin{array}{l}\text { Know } \\
\text { n(\%) }\end{array}$ & $\begin{array}{l}\text { Don't } \\
\text { Know } \\
\text { n(\%) }\end{array}$ \\
\hline $246(93.2)$ & $18(6.8)$ \\
\hline $201(76.2)$ & $63(23.8)$ \\
\hline $206(78.0)$ & $58(22.0)$ \\
\hline $201(76.0)$ & $63(24.0)$ \\
\hline $168(63.6)$ & $96(36.4)$ \\
\hline $130(49.2)$ & $134(50.8)$ \\
\hline $147(55.7)$ & $117(44.3)$ \\
\hline $185(70.0)$ & $79(30.0)$ \\
\hline $130(49.2)$ & $134(50.8)$ \\
\hline $134(50.8)$ & $130(49.2)$ \\
\hline $153(58.0)$ & $111(42.0)$ \\
\hline $221(83.7)$ & $43(16.3)$ \\
\hline
\end{tabular}

1. DM patients should take medication regularly because they are liable to get DM complication.

2. DM patients should look after their feet because they may not feel a minor injury to their feet.

3. DM patients should look after their feet because wounds and infection may not heal quickly.

4. DM patients should look after their feet because they may get a foot ulcer

5. DM patients should not smoke because smoking causes poor circulation and affects the feet.

6. Do you know how often do you think you should inspect your feet?

7. If you found redness/bleeding between your toes what is the first thing you do?

8. How often do you think your feet should be washed?

9. What temperature of water do you think you should wash your feet in?

10. How often do you think you should inspect the inside of your footwear for objects or torn lining?

11. How often do you think you should wear shoes and socks?

12. Do you think you can avoid foot problems if keep your blood glucose 221(83.7) 43(16.3) level is controlled?

Table 2: Percentage distribution of responses regarding self- foot care practice .

.No. Questions related to foot care practice

1. Do you examine/inspect your feet regularly?

2. Do you wash feet regularly? Not done 1.

3. Do you wash feet with warm water?

147(55.7) $117(44.3)$

4. Do you receive advice when last you bought footwear?

219(83.0) 45(17.0)

5. Do you regularly check the water temperature before use?

154(58.3) $110(41.7)$

6. Did you ever inspect inside of footwear?

$108(41.0)$ $156(59.0)$

7. Do you add irritants to water before feet cleaning?

103(39.0)

$161(61.0)$

8. Do you regularly Dry between toes?

144(54.5)

$120(45.5)$

9. Do you trim toe nails straight across?

$70(26.5)$

194(73.5)

10. Do you clean nails with sharp instrument?

$141(53.4)$

94(33.6)

$109(41.3)$

123(46.6)

11. Do you add Talcum powder to the interdigital space to keep them dry?

$74(28.0)$

$155(58.7)$

12. Do you add skin emollient or cream to the bottom or the top of your feet to keep them smooth?

13. Do you check your shoes before you put them on?

\begin{tabular}{l|l}
$100(38.0) \quad 164(62.0)$
\end{tabular}

14. Do you check your shoes when you take them off?

136(51.5) $128(48.5)$

15. Do you regularly walk bare foot inside the house?

16. Do you regularly walk bare foot outside the house?

$104(39.4)$

$160(60.6)$

$133(50.4)$

$131(49.6)$

17. Do you remove callosities and rough skin yourself?

$84(31.8)$

$180(68.2)$

109(41.3)

155(58.7)

than those without, and patients with good foot self-care knowledge level were ten times more likely to practice foot care as compared to poorly knowledgeable patients $(\mathrm{p}<0.001)$.

\section{Discussion}

Diabetic foot ulcer is one of the chronic complications of DM in which patients end up with disability and death if it is not 
Table 3 : Regression analysis model for diabetic self- foot care practice.

\begin{tabular}{|l|l|l|l|l|l|}
\hline Variable & $\mathbf{B}$ & Wald & $\mathbf{p}$-value & $\mathbf{E x p}(\mathbf{B})$ & CI (95\%) \\
\hline Age & 0.05 & 0.84 & 0.3 & 1.05 & $(0.9-1.17)$ \\
\hline Duration of DM & -0.071 & 1.13 & 0.2 & 0.93 & $(0.8-1)$ \\
\hline Age Onset & -0.084 & 2.45 & 0.11 & 0.91 & $(0.8-1.02)$ \\
\hline Sex : Male & 0.43 & 0.33 & 0.56 & 1.5 & $(0.35-6.7)$ \\
\hline Residence :Urban & -0.37 & 0.54 & 0.4 & 0.68 & $(0.25-1.8)$ \\
\hline Income :Not enough & -0.7 & 0.82 & 0.3 & 0.46 & $(0.086-2.4)$ \\
\hline Education :Low & -0.057 & 0.004 & 0.9 & 1.05 & $(0.19-5.9)$ \\
\hline $\begin{array}{l}\text { Type of DMType I } \\
\text { History of DFU* +ve } \\
\text { history }\end{array}$ & 1.04 & 2.67 & 0.1 & 2.8 & $(0.8-10)$ \\
\hline $\begin{array}{l}\text { Chronic disease: } \\
\text { Presence }\end{array}$ & 1.02 & 3.65 & 0.05 & 2.7 & $(1.02-10.8)$ \\
\hline $\begin{array}{l}\text { Knowledge level* } \\
\text { Good level }\end{array}$ & 22.4 & 40.2 & $0.0001^{*}$ & 10.4 & $(0.97-7.9)$ \\
\hline
\end{tabular}

$* \mathbf{p}<0.05$

effectively prevented and controlled. ${ }^{6}$ Which contribute substantially to the health care costs associated with diabetes. ${ }^{9}$ In this study, out of 264 study subjects ,about $34.1 \%$ and $29 \%$ of the respondents' age were between (45-55), (56-65) years age group respectively, because the majority of studied patients(77\%) suffered from type II DM and this is the age onset of type II DM. This observation contradicts with an Ethiopian study, because the majority of its respondents' ages were much younger $(18-25)$ years, as most of them had type I DM. ${ }^{6}$

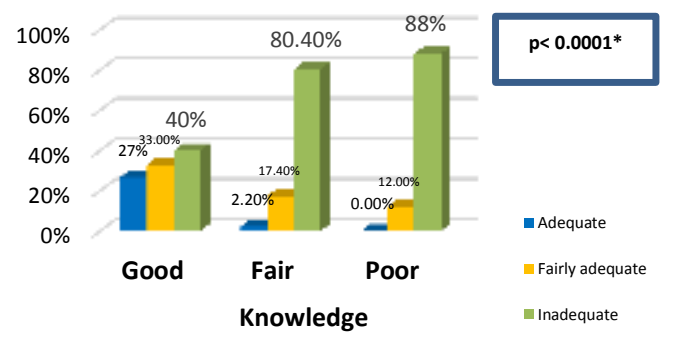

Figure 1: Relationship between diabetic selffoot care knowledge and practice among the studied patients.

According to American Diabetic Association 2016, nutrition therapy is a component of overall treatment for type I and II diabetes as eating pattern improve glucose, blood pressure and lipids. ${ }^{11}$ Only $3 \%$ of the studied patients follow the recommended diet regimen for DM, this could explain the poor glycemic control found between them.

The poor compliance of patients regarding diet control could be explained by Pavan Kumar Jain, that patients especially in developing countries have poor knowledge

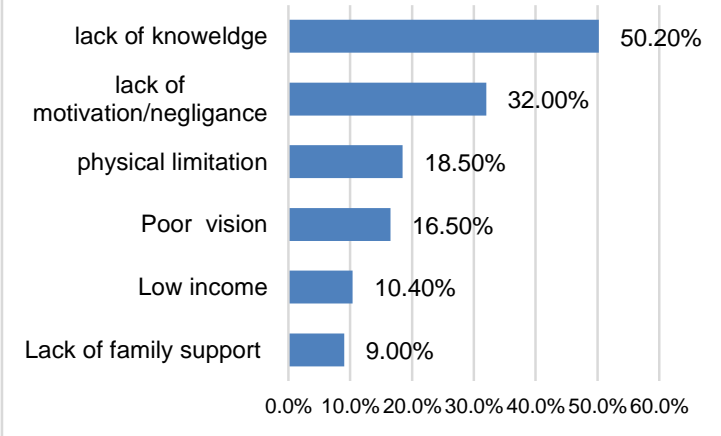

Figure 2: Perceived barriers against adequate self-foot care.

regarding caloric requirements, food exchange list and sources. Thus, nutritional education is a necessitate for diabetic patients. ${ }^{20}$ 
History of developing diabetic foot ulcer was $(28.0 \%)$, this result was in the same line with others' findings ;in Taiwan $(29.3 \%)^{21}$, in the UK and USA $19.0 \%^{22}$, and in Egypt $32.4 \%{ }^{4}$ More than half of participants had neuropathy (56\%) (Table 2 ), which is a common risk factor of diabetic foot ulcer formation besides trauma and foot deformity. ${ }^{2,3}$

The study results on knowledge of principles of diabetic foot self-care indicates that the mean knowledge score was $8 \pm 3.1$ which is higher than previous studies ,done in Nigeria in which the score was $5.8 \% \pm 3.3,{ }^{24}$ and Ethiopia $7.5 \pm 2.02 .{ }^{6}$

The responses for each knowledge measure of foot care were generally good except that, half of the respondents didn't know how often they should inspect their feet or the foot wear and what is the suitable temperature of water, they should wash their feet in, this is higher than other two related studies in Ethiopia (32\%) and India $(16.4 \%) .{ }^{6,7}$ The possible cause for this difference could be better education opportunities and socioeconomic conditions in Egypt.

Half of the participants had good knowledge level (51.3\%) similar to a study conducted in Eithiopia. ${ }^{6}$ Poor knowledge level was $(31.4 \%)$, considered to be lower compared to other studies in Egypt (39\%) ${ }^{4}$, Nigeria $(46 \%)^{24}$, Malaysia $(58.0 \%)^{25}$, and in Gujarat $(62.54 \%){ }^{20}$

As regard overall practice score of studied patients, the current findings show that more than half $(62.2 \%)$ of them were not adequately cared for their feet inspite of their knowledge. Consistently with Awoel et al 2015 and Sanaa et al., 2016 findings, in which $45.4 \%$ and $57.8 \%$ respectively had poor practice for diabetic foot selfcare. $^{6,4}$

Based on the specific measures of foot care practice, the ones that received poorest responses were: checking the temperature of water before using; applying talcum powder in the interdigital space, those observations goes with poor practices detected among Malaysian diabetic patients. 25

Checking the temperature of water before using is an important step that should be taught to patients, so that scald injury could be avoided especially in the feet of diabetics, as diabetic patients with neuropathy might not be able to feel any insult to their feet. Patient should not use water that is too hot or too cold in washing and bathing. Keeping the interdigital space dry by applying talcum is also important to prevent fungal infections as a part foot care hygiene. ${ }^{26}$

As DFU may result from fissures and cracks, which occur frequently in diabetic patients with autonomic neuropathy. ${ }^{27}$ Thus, the practice of applying lotion is suggested for treating the dry skin of the feet, ${ }^{28}$ but more than half of patients $(62.0 \%)$ didn't add skin emollient or lotion to their feet in order to keep them hydrated and smooth.

Although the majority of study participants have never walked barefoot outdoors, still the proportion of respondents walking barefoot outdoors is not negligible $(31.8 \%)$, besides half of respondents routinely walk barefoot indoors which is considered as major risk behavior among the studied patients.

Those proportions are similar with a study in South Africa, 25\% walked barefoot indoors and outdoors ${ }^{(9)}$. While it is higher than in Ethiopia in which $15.3 \%$ of study participants walk barefoot indoors and $9.6 \%$ in outdoors. $^{6}$

It is necessary to pay attention to the importance of foot drying, whereas, most of Egyptians are Muslims, they pray five times per day where the feet have to be washed. However, washing feet and the 
praying itself offer some sort of physical massage to the feet. Also, trimming nails is a habit encouraged by Islam. ${ }^{14}$

Regarding to sociodemographic characteristics, gender had no role in determining the level of knowledge and practice of diabetic foot care in the present study. However, two studies conducted in Europe and Malaysia reported that women have a significantly higher knowledge and practice score compared to men. ${ }^{18,25}$

An important finding of this study, was a significant association between level of education with the level of diabetic foot care knowledge and practice $(\mathrm{p}=0.001,0.02$ respectively), this in line with several studies that looked into knowledge and practice of diabetic foot care. ${ }^{20,24,29-31}$

Meaning that education may lead to awareness of diabetic self-foot care principles, which is the basic preventive measure of foot ulceration. However, knowledge of the foot care alone is not helpful unless practiced.

Interestingly, the score of practice was poorer than the score of knowledge, where only $14.0 \%$ of patients had good practice level compared to $51.0 \%$ had good knowledge level. This observation indicates poor patients compliance; patients had some degree of foot care awareness but they didn't practice what they know. This finding agreed with previous studies, that reported the same pattern for knowledge and practice of foot care. $^{24,25,29,31}$

Inadequate practice was significantly found among type II diabetic patients more, this may argued that most of type I patients pay more attention to practice ,because of their younger age and they may have poly-symptoms, so that they insist to seek medical care early compared to type II patients. ${ }^{6}$

Physicians have an elementary role in improving awareness and adequate practices of foot care among their patients. Insufficient communication between healthcare providers and patients with limited time devoted to educate patients as a result of busy clinic schedule are the main cause of inappropriate patient education. ${ }^{19,31,32}$

As for the medical care provided by the health professionals, $168(63.8 \%)$ patients reported they weren't guided by the nurses nor the physicians about foot self-care principals. Indicating that there was improper health education of patients, a previous study also reported the same observation. ${ }^{6}$ Therefore, physicians should always be aware with the recent guidelines of foot care and ensure the proper compliance of their patients.

The common barrier of diabetic foot care mentioned by respondents was: "poor communication between patients and health care providers (54.7\%) (Figure 2). That is agree with barriers reported in Awole Seid1 and Yosief Tsige study "(56.8\%). ${ }^{6}$

On the other hand, half of patients reported lack of adequate knowledge to specific diabetic foot care principles as another barrier of foot care which could be attributed to the poor communication between patients and health care providers. Previous studies also reported similarly. ${ }^{6,24,33}$

Finally, our findings unveiled the unavoidable barrier of limited access to podiatry care services, which is a prevalent issue in most of developing communities. ${ }^{34}$ In Egypt, there are no well-defined policies or programs in educating patients within inpatients or outpatients settings. ${ }^{4}$

\section{Conclusion}

Knowledge of foot care in the majority of diabetic patients was good, while the practice was inadequate. Health care personnel have an elementary role in 
promoting proper diabetic patients foot self-care. Educational programs aiming to improve the awareness of foot care should be performed from primary care level up to tertiary care centers as a multidisciplinary team approach.

\section{Conflict of Interest}

Author (1) Mira Maged Mohamed Abuelenin:There was no conflict of interest

Author (2) Ahmed Abdel Fattah Elshora :There was no conflict of interest

Author (3) Ghada Mahmoud Atia. Alghazaly: There was no conflict of interest

Financial disclosure: No financial disclosures were reported by the authors of this paper.

\section{References}

1. Driver V, Fabb0i M, Lavery L, Gibbons G. The Cost of Diabetic Foot: The Economic Case for the Limb Salvage Team. J Vasc Surg. Dec 2010; 52(6):1751.

2. Armstrong DG, Cohen K, Courric S, Bharara M.,Marston W. Diabetic foot ulcers and vascular insufficiency: our population has changed, but our methods have not.Journal of Diabetes Science and Technology.2008; 5(6): 1591-1595.

3. Yahia MK.Foot care among male diabetics in family practice center of Saudi Arabia.Journal of Family and Community Medicine. 2008; 15 (3):103-106.

4. Sanaa AE, Mekkawy MM, Besely WF et al. Prevalence of Risk Factors for Egyptian Diabetic Foot Ulceration .Journal of Nursing and Health Science.2016; 5(2): 45-57.

5. K. Alexiadou and Doupis J .Management of diabetic foot ulcers. DiabetesTherapy.2012; $3(4): 4$.

6. Seid AW and Tsige YO .Knowledge, Practice, and Barriers of Foot Care among Diabetic Patients Attending Felege Hiwot Referral Hospital,Bahir Dar, Northwest Ethiopia, Advances in Nursing.2015; 2015: 19. Article ID 934623.

7. Aggarwal SA, Bhardwaj VI, Singh AB. et al. Self Reported Perception Towards
Diabetic Foot Care Among Patients Seeking Care At Tertiary Care Teaching Hospital In Rural Haryana. Indian J.Sci.Res.2015; 6(2) : 49-54.

8. Lipsky BA, Weigelt JA, Sun X, Johannes RS, Derby KJ. Developing and validating a risk score for lower-extremity amputation in patients hospitalized for a diabetic foot infection. Diabetes Care.2011;34(8):1695-1700.

9. Amogne W, Reja A, Amare A. Diabetic foot disease in Ethiopian patients: a hospital based study.Ethiopian Journal ofHealth Development.2011;25(1): 17-21.

10. Allen L, Park N, Diehl K, Driver V. Limb Loss and the Effect of a Multidisciplinary Treatment Approach on Global Populations: A team approach can reduce diabetic morbidity globally. Available at: www.podiatrym.com 2014. Accessed on August 172016.

11. Miller J , Carter E, Shih J et al. How to do a 3-minute diabetic foot exam; 2014 available at: www.jfponline.com/diabetes/how-to-do-a3-minute .Accessed on June 252016.

12. Centers for Disease Control and Prevention . National diabetes fact sheet: national estimates and general information on diabetes and pre-diabetes in the United States, 2011. Atlanta. Department of Health and Human Services In: Prevention The diabetic foot examination: A positive step in the prevention of diabetic foot ulcers and amputation. Osteopathic Family Physician .2013; 5:73-78.

13. Li RZ, Barker LE, Chowdhury FM et al. Cost-Effectiveness of Interventions to Prevent and Control Diabetes Mellitus: A Systematic Review. Diabetes Care.2010; 33(8):18721894, 1188-119.

14. Ahmed AA, Elsharief EM, Alsharief AL. The Diabetic Foot in the Arab World. The Journal of Diabetic Foot Complications. 2011; 3(3):55-61.

15. MENA 2016.International federation of diabetes available at https://www.idf.org/ournetwork/regions-members/middle-east-andnorth-africa/welcome. Accessed July 152016. 16. International Diabetes Federation. IDF Diabetes Atlas, 6th edition. Brussels, 2013. 
Available at https://www.idf.org/e library/epidemiology-research/diabetesatlas.html. Accessed August 202016.
17. American
Diabetes
Association

(ADA):Standards of medical care in diabetes 2016. Diabetes Care. 2016;39(1):S1-S106.

18. Pollock RD, Unwin NC, Connolly V. Knowledge and practice of foot care in people with diabetes. Diabetes Res Clin Pract. 2004; 64(2): 117-22.

19. Guell CO,Unwin NI. Barriers to diabetic foot care in a developing country with a high incidence of diabetes related amputations: anexploratory qualitative interview study BMC Health Services Research. 2015;15:377.

20. Pavan KJ. Knowledge \& Attitude of Diabetic Patients Regarding Diabetic diet, Exercise and Foot care. International Journal of Nursing Education. 2012; 4(2):141-145.

21. Chin YF, Liang J, Wang WS et al.The role of foot self-care behavior on developing foot ulcers in diabetic patients with peripheral neuropathy: a prospective study. Int J Nurs Stud. 2014 Dec;51(12):1568-74. doi: 10.1016/j.ijnurstu.2014.05.001.

22. Gonzalez J.S,. Vileikyte L ,Ulbrecht J.S, Rubin R.R.,Depression predicts first but not recurrent diabetic foot ulcers. Diabetologia .October 2010; 53(10) : 2241-2248.

23. Al.Rubeaan KH, Al Derwish MA, Ouizi SA, et al., Diabetic Foot Complications and Their Risk Factors from a Large Retrospective Cohort Study.PLoS One. 2015; 10(5]: e0124446.

24. Desalu OO, Salawu FK, Jimoh AK, Adekoya AO, Busari OA. Diabetic foot care: self reported knowledge and practice among patients attending three tertiary hospital in Nigeria.Ghana Medical Journal.2011; 45(2): 60-65.

25. Muhammad-Lutfi AR, Zaraihah MR, Anuar-Ramdhan IM, Knowledge and Practice of Diabetic Foot Care in an In-Patient Setting at a Tertiary Medical Center.Malaysian Orthopaedic Journal 2014;8(3):22-26.

26. Mason J, O'Keefe CO, Mcintosh A, et al . A Systemic Review of Foot Ulcer in Patients with type2 Diabetes Mellitus. I: Prevention. Diabet Med. 1999; 16(10): 801-12.

27. Oe, M , Sanada H, Nagase TM. Factors associated with deep foot fissures in diabetic patients: a cross-sectional observational study. Int. J. Nurs. Stud. 2012;49 (6) :739-746.

28. Bakker K, Apelqvist, J, Schaper, NC., the International Working Group on the Diabetic Foot Editorial Board, 2012. Practical guidelines on the management and prevention of the diabetic foot 2011. Diabetes Metab. 2011; 28 (1) 225-231.

29. .Hasnain S, Sheikh NH. Knowledge and practices regarding foot care in diabetic patients visiting diabetic clinic in Jinnah Hospital, Lahore. J Pak Med Assoc. 2009; 59(10): 687-90 .

30. .Lamchahab FZ, El Kihal N, Khoudri I et al..Factors influencing the awareness of diabetic foot risks. Ann Phys Rehabil Med. 2011; 54(6): 359-65.

31. Chellan G, Srikumar S, Varma AK et al.. Foot care practice - The key to prevent diabetic foot ulcers in India. Foot (Edinb). 2012; 22(4): 298-302.

32. Ang CL and Lim YJ. Recurrent Admissions for Diabetic Foot Complications. Mal Orthop J. 2013; 7: 21-6.

33. Bengalorkar GM and Nagendra KT. Diabetic foot infections-a review," International Journal of Biological and Medical Research.2011; 2(1): 453- 460.

34. Unwin $\mathrm{N}$. The diabetic foot in the developing world. Diabetes Metab Res Rev. 2008; 24 (1):S31-33. 